\title{
De la escrófula a la linfoadenitis micobacteriana
}

\author{
Ignacio Duarte
}

\section{From scrophula to mycobacterial lymphadenitis}

In the $19^{\text {th }}$ century it was widely believed that both tuberculosis and cervical lymph node swelling, known as scrophula, affected individuals predisposed to an inherited "diathetic constitution". In 1882 Robert Koch proved that human tuberculosis and scrophulous lesions were caused by the bacillus Mycobacterium tuberculosis. In the early twentieth century it was stated that Mycobacterium bovis, the bacillus of cattle tuberculosis, could also cause cervical lymphoadenitis in humans, especially in children, by the intake of milk from sick cows. The incidence of this condition decreased after the infection was controlled in cattle and pasteurization of the milk was introduced. A type of granulomatous necrotizing and suppurative cervico-facial lymphadenitis associated to non-tuberculous mycobacteria was described in 1956. It mainly affects children younger than 5 years old, particularly those born in countries with non-endemic tuberculosis. Tuberculous cervical lymphadenitis is prevalent in young adults from tuberculosis-endemic countries and in HIV-infected subjects. Infectious etiology displaced the importance of a personal disposition in the development of scrophula. Nevertheless, mutations that confer susceptibility to mycobacterial infection are currently investigated.

Key words: Scrophula, lymphadenitis, tuberculosis, mycobacteria, cattle, pasteurization.

Palabras clave: Escrófula, linfoadenitis, tuberculosis, micobacterias, ganado, pasteurización.
Pontificia Universidad Católica de Chile. Santiago, Chile. Programa de Estudios Médicos Humanísticos. Facultad de Medicina.

Recibido: 3 de octubre de 2017

Correspondencia a: Ignacio Duarte G. ignacioduarte.gc@gmail.com

\section{Introducción}

$\mathrm{E}$ n el siglo XIX era frecuente la escrófula, llamada también adenitis escrofulosa o adenitis caseosa. Su prototipo era la tumefacción crónica de los ganglios linfáticos cervicales con tendencia al reblandecimiento y fistulización; pero también podía afectar otros sitios del organismo. Esto dio origen a diferentes opiniones acerca de su naturaleza, causas y especialmente su relación con la tuberculosis o tisis pulmonar que era frecuentemente consuntiva y letal. Se discutía sobre si ambas afecciones eran manifestaciones de una sola enfermedad o se trataba de dos entidades distintas.

Predominaba la idea de que ambos cuadros clínicos estaban condicionados por una diátesis hereditaria sobre la cual actuarían agentes desencadenantes derivados del ambiente, costumbres o excesos ${ }^{1}$. No parecía tener muchos adherentes la posibilidad de un agente específico transmisible. En la segunda mitad de la centuria, el desarrollo de la microbiología demostró que gérmenes microscópicos podían causar enfermedades en los seres humanos, afianzándose la teoría del contagio en la etiología de diversos males. El objeto de esta publicación es indagar la evolución de las ideas acerca de la naturaleza y caracteres de la enfermedad escrofulosa desde fines del siglo XIX.

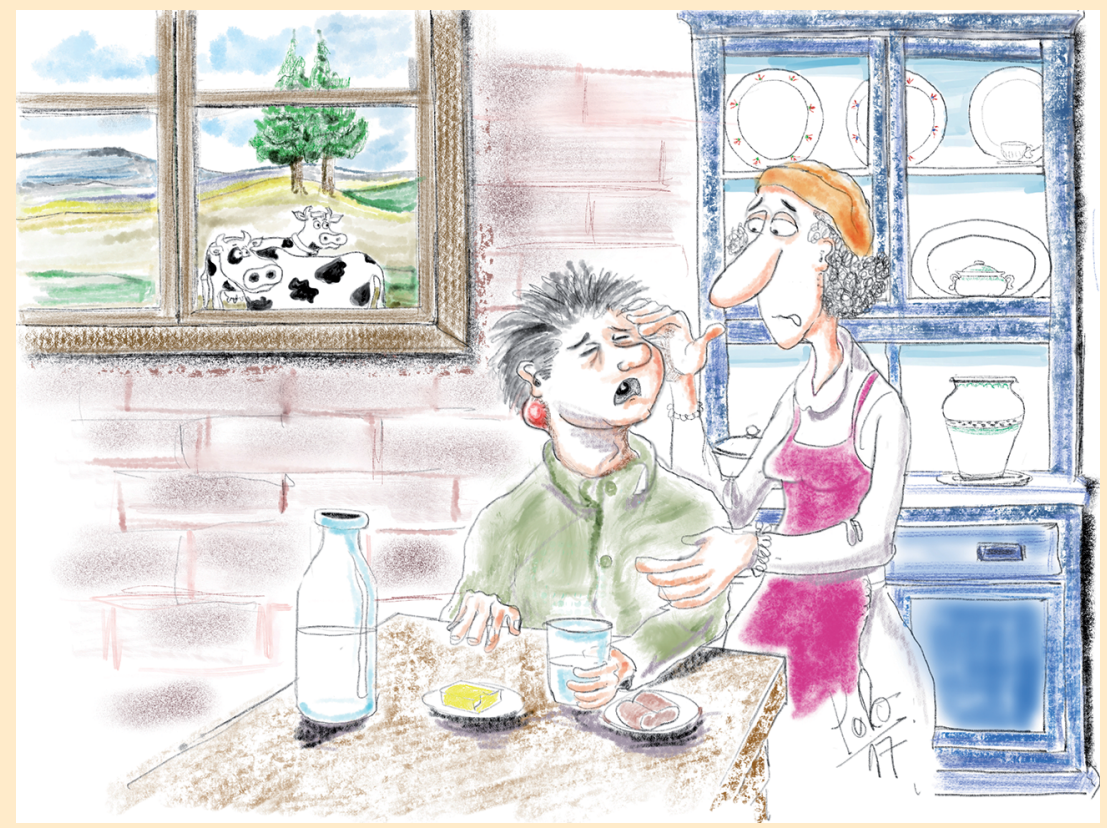

\section{El descubrimiento de la etiología de la tuberculosis}

En 1882 Robert Koch comunicó los resultados de sus investigaciones sobre la etiología infecciosa de la tuberculosis. Examinó al microscopio tejidos provenientes de 
33 casos humanos de tuberculosis de diversos órganos. Con tinción de azul de metileno, contrastada con pardo Bismarck en frotis y cortes histológicos de las lesiones, vio bacilos teñidos muy nítidamente de color azul, en contraste con el resto del tejido y con bacterias no tuberculosas, que se teñían de color café. Tanto por sus hallazgos microscópicos como por cultivo e inoculación en animales pudo proponer que, en lo sucesivo, el diagnóstico de tuberculosis no se basaría en el cuadro histopatológico, sino por la demostración del bacilo por tinción en los tejidos o por cultivo. En este sentido, afirmó que afecciones como la tuberculosis miliar, la neumonía caseosa, la bronquitis caseosa y la tuberculosis intestinal podían ser declaradas idénticas por el hallazgo de los bacilos en esos casos ${ }^{2}$.

Entre las muestras humanas que Koch examinó figuraban tres trozos de ganglios escrofulosos recién extraídos: en dos de ellos demostró bacilos positivos con azul de metileno. También pudo cultivar bacilos tuberculosos a partir de dos muestras de escrófula humana. Frente a este resultado, Koch estimó que el número de casos estudiados no permitía una clara decisión sobre su etiología, aunque pensaba que un gran número de ganglios linfáticos escrofulosos correspondían a verdadera tuberculosis ${ }^{2}$.

La noticia de los hallazgos de Koch se diseminó y acogió rápidamente, con la resistencia de algunos personajes que no creían en la teoría de los gérmenes, o bien que no habían podido replicar los hallazgos de Koch por errores técnicos ${ }^{3}$. Se extendió la práctica diagnóstica de identificar bajo el microscopio los bacilos tuberculosos, que después de teñidos tenían la propiedad de resistir a la decoloración con una combinación de ácido y alcohol ${ }^{4,5}$. Por esta razón se les empezó a reconocer como "bacilos ácido-alcohol resistentes" (Figura 1).

Un calificado testigo y protagonista de la evolución de las ideas sobre la tuberculosis durante la transición entre los siglos XIX y XX fue el médico Edward Livingston Trudeau, que nació en Nueva York en 1848. En 1865 su hermano sufrió una tuberculosis pulmonar que progresó rápidamente a la muerte. Edward cuidó al enfermo, con quien compartía la habitación, la cual, según los médicos, debía estar con las ventanas permanentemente cerradas ${ }^{6}$.

Trudeau inició sus estudios médicos en 1868; su profesor enseñaba que la tuberculosis era "una enfermedad no contagiosa, debida a peculiaridades constitucionales heredadas, alteraciones de los humores y variados tipos de inflamación, que conducían al desarrollo de tubérculos, escrófula, caseificación y tisis pulmonar"6.

Cuando cursaba el segundo año, a raíz de sufrir un malestar general, consultó un médico que encontró un absceso frío - cuya ubicación no se menciona- que fue atribuido a consecuencia del esfuerzo de una caminata reciente. El absceso tuvo que ser operado varias veces antes que sanara.

Después de titularse en 1871 notó una tumefacción de los ganglios laterales del cuello. Un prestigioso médico le explicó que la hinchazón ganglionar era un signo de debilidad con tendencia a la escrófula. Le recetó un tónico a base de hierro, aplicaciones locales de yodo y le aconsejó desayunar con abundante tocino. En la autobiografía no hay información sobre la evolución de aquel cuadro clínico.

Trudeau ejerció la profesión en Estados Unidos. En 1873 se le diagnosticó tuberculosis activa de los dos tercios superiores del pulmón izquierdo. En su autobiografía refiere que ni con la primera advertencia (absceso frío) ni con la segunda (tumefacción cervical) se había percatado de que estaba afectado con la misma enfermedad letal de su hermano. Pensando que pronto iba a morir se trasladó a la región de los montes Adirondack en el estado de Nueva York, donde llevó un régimen de reposo y, a medida de sus fuerzas, dedicarse a una vida más activa al aire libre. Paulatinamente se fue sintiendo mejor, aunque sufría recrudecimientos. Se estableció definitivamente en un pueblo de la región a orillas del Lago Saranac. Empezó a dar atención médica a personas que iban a pescar o a cazar, y también a tuberculosos en plan de reposo. Inspirado en la experiencia de los primeros sanatorios para tuberculosos en Europa, Trudeau consiguió donaciones y creó en 1884 el Adirondack Cottage Sanitarium de Saranac, formado por pequeñas cabañas independientes para el reposo de tuberculosos de recursos modestos ${ }^{6}$.

Trudeau quedó impresionado cuando leyó una traducción del artículo original de Koch. Aprendió en Nueva York las técnicas elementales de bacteriología y montó en Saranac un modesto laboratorio donde teñía el bacilo tuberculoso, aplicando el método al examen del esputo de los pacientes. También logró cultivarlo, lo que le permitió realizar experimentos sobre métodos de tratamiento, la

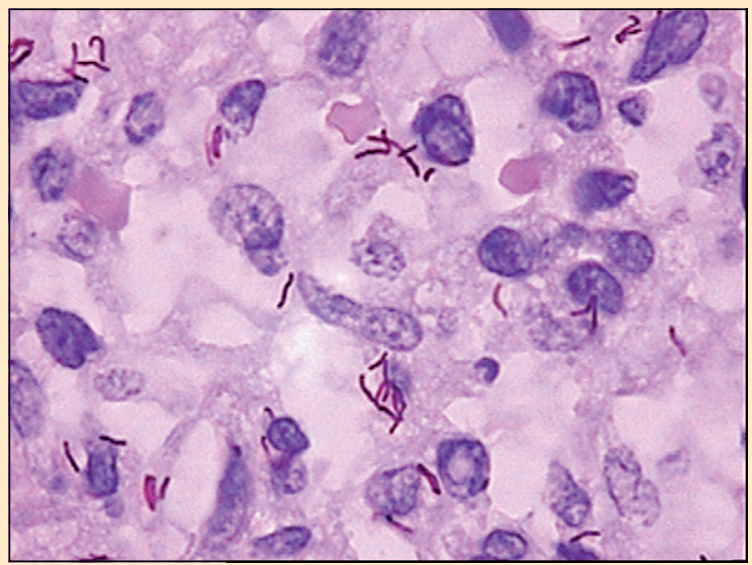

Figura 1. Corte histológico a gran aumento de una lesión tuberculosa. Destacan los bacilos ácido-alcohol resistentes sobre el fondo pálido (Tinción de Ziehl-Neelsen). 
influencia del ambiente en el desarrollo de la tuberculosis, y las posibilidades de inducir inmunidad artificial, publicando más de una cincuentena de artículos ${ }^{7,8}$. Fue elegido primer presidente de la Asociación Nacional para el Estudio y Prevención de la Tuberculosis y recibió distinciones de diversas instituciones. Continuó trabajando con intermitencias debido a su enfermedad, falleciendo de tuberculosis en $1915^{7}$.

\section{Linfoadenitis tuberculosa}

Los términos escrófula, adenitis estrumosa o caseosa se fueron reemplazando en el siglo XX por el de linfoadenitis tuberculosa. En la década de 1930 se consideraba la escrófula de los niños como una forma especial de la tuberculosis que afectaba principalmente los ganglios cervicales, mencionándose todavía la importancia del estado tímico-linfático como factor predisponente. El estudio histopatológico de los linfonodos afectados mostraba necrosis caseosa y granulomas epiteloideos con células gigantes multinucleadas, que correspondían a los tubérculos caracterizados por Virchow (Figuras 2 y 3$)^{9-11}$. Se explicaba que los ganglios cervicales se infectan por vía linfática a partir de las amígdalas (tuberculosis descendente) o por la propagación de la lesión de los ganglios linfáticos bronquiales (tuberculosis ascendente), a su vez proveniente del compromiso pulmonar ${ }^{10}$. Se agregaba que rara vez la infección alcanzaba los ganglios linfáticos por vía sanguínea ${ }^{12}$. En 1958 Miller y Cashman comunicaron una serie de niños con diagnóstico clínico de linfoadenitis tuberculosa periférica y concluyeron que a lo menos $80 \%$ de los casos representaban el componente ganglionar de un complejo primario en una zona vecina. Los grupos ganglionares más frecuentemente comprometidos eran los submentonianos y el yugulodigástrico o tonsilar en 118 casos: en 91 la adenitis cervical era el único signo de tuberculosis, descartada en otros sitios con examen clínico y radiológico de cuello, tórax y abdomen. En 15 pacientes encontraron un foco primario en boca o amígdala. En algunos casos de compromiso de ganglios preauriculares describieron focos primarios en piel de la frente o mejillas, en la conjuntiva palpebral o en el saco lacrimal. No se mencionaban exámenes microbiológicos ${ }^{13}$.

\section{Linfoadenitis por bacilo bovino}

Lehmann y Neumann propusieron en 1896 el nombre Mycobacterium para el género que comprendía tres especies: M. tuberculosis, Mycobacterium tuberculosis avium y Mycobacterium leprae ${ }^{14}$. En 1898 el microbiólogo estadounidense Theobald Smith estudió bacilos tuberculosos obtenidos de esputos de pacientes ( $M$.

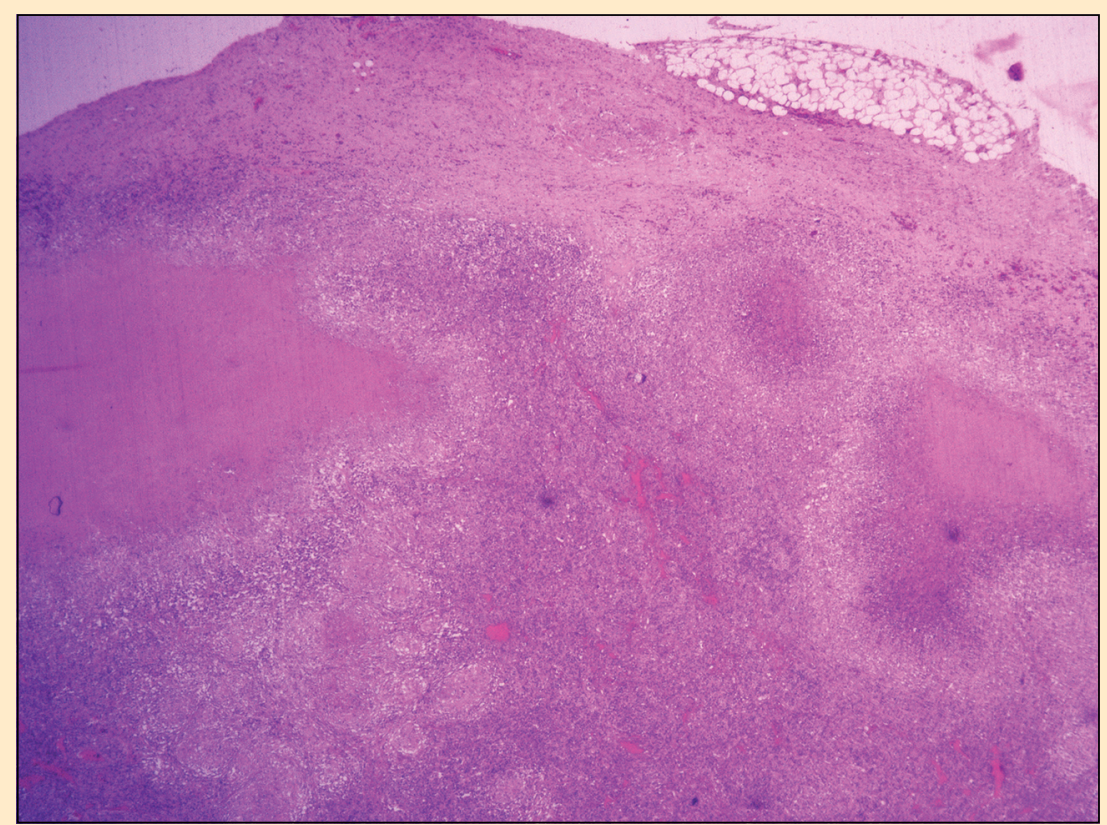

Figura 2. Corte histológico de ganglio linfático, a bajo aumento. Está delimitado arriba por una cápsula. Debajo, estructura ganglionar distorsionada por formaciones irregulares con centro rosado (necrosis caseosa) rodeado por una banda pálida (conjunto de células epiteloideas). Hacia abajo, formaciones redondeadas rosadas pálidas pequeñas que corresponden a tubérculos (granulomas tuberculosos) (Tinción hematoxilina-eosina).

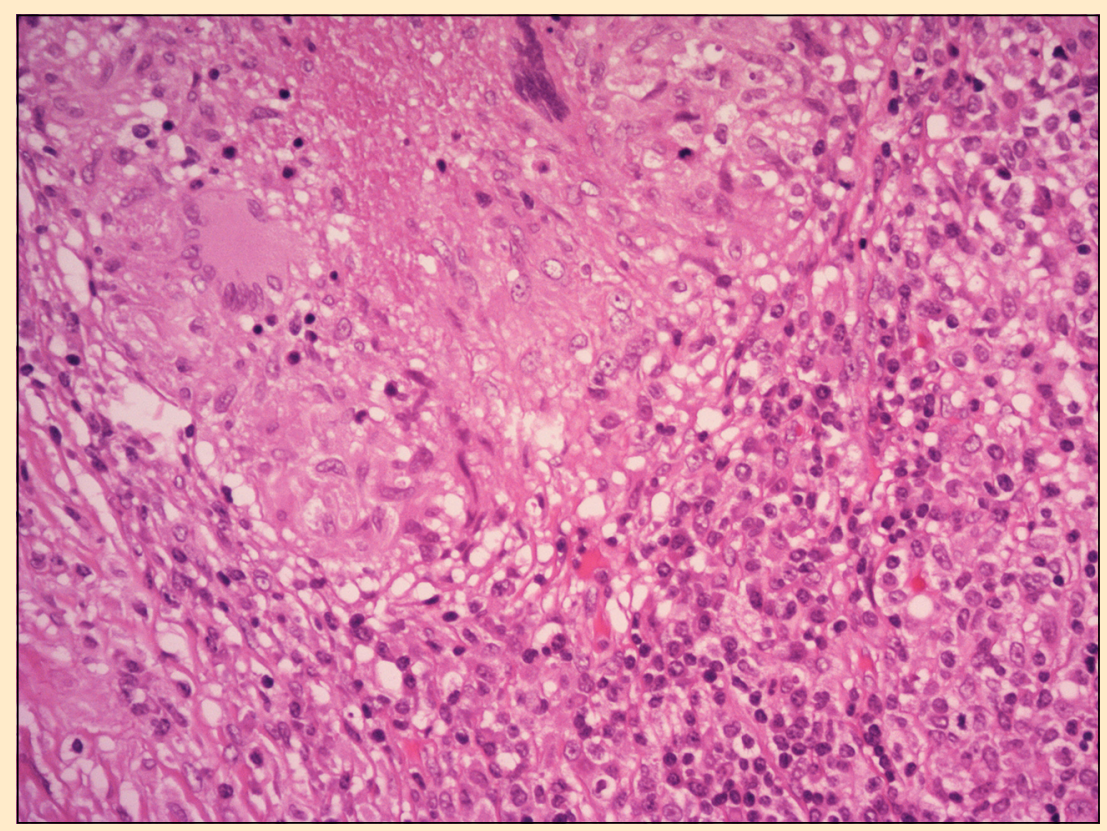

Figura 3. Corte histológico de un ganglio linfático, a mayor aumento. En la zona superior izquierda, un tubérculo de contorno redondeado, constituido por células epiteloideas. Se reconocen dos células gigantes multinucleadas: la situada a la izquierda, posee núcleos dispuestos en semicírculo (célula de Langhans). En el centro del tubérculo, pequeña área rosada donde no se reconocen núcleos: corresponde a necrosis (Tinción hematoxilina-eosina). 
tuberculosis), comparándolos con bacilos de lesiones tuberculosas de ganado bovino, principalmente vacas. Describió diferencias entre ambos grupos tanto en los caracteres de los cultivos como en los experimentos de inoculación en animales. Sugirió que la ingestión de leche de vaca podía producir en los niños una tuberculosis por vía digestiva ${ }^{15}$. La discusión acerca de si el bacilo bovino podía causar enfermedad en el ser humano fue resuelta a base de diversos datos, principalmente los aportados en 1911 por la Royal Commission on Tuberculosis inglesa: mediante inoculación con bacilo bovino se concluyó que una vaca tuberculosa podía eliminar bacilos en la leche, especialmente si tenía una lesión tuberculosa en las ubres; pero también la leche podía contaminarse con las heces o descargas uterinas de la vaca enferma, después de ordeñada ${ }^{16}$. El nombre Mycobacterium bovis para el bacilo del ganado se habría propuesto alrededor de $1930^{17}$. En la primera mitad del siglo XX se comunicaba que 0,6 a $2 \%$ de los casos de tuberculosis pulmonar eran causados por el bacilo bovino, presumiblemente por vía respiratoria en personas expuestas al ambiente de los establos. En un cuarto a un tercio de aquellos casos había antecedente de compromiso ganglionar cervical o abdominal, óseo o $\operatorname{articular}^{18}$. Una compilación de series británicas indicaba que alrededor de la mitad de los casos de tuberculosis ganglionar cervical se debía al bacilo bovino, proporción que alcanzaba hasta $90 \%$ en pacientes bajo los cinco años ${ }^{19}$. Por otra parte, la comparación del hallazgo de bacilo bovino y de bacilo humano mostraba que en menores de 16 años la proporción de bacilo bovino era de $2 \%$ en tuberculosis pulmonar y de ganglios bronquiales, $34 \%$ en ganglios cervicales y axilares, $40 \%$ en "órganos abdominales", y $29 \%$ en huesos y articulaciones. La proporción de casos con bacilo bovino era menor en sujetos mayores de 16 años ${ }^{20}$.

Los datos mencionados apuntaban a que la tuberculosis ganglionar era más frecuente en niños, que se alimentaban principalmente de leche, y que era causada por bacilo bovino en una proporción sustancial de los casos. En ciudades europeas se encontraba el bacilo bovino en 15 a $33 \%$ de las leches comerciales examinadas ${ }^{21}$. Dado que era frecuente la tuberculosis en el ganado vacuno, se apreció la necesidad de controlarla detectando la enfermedad, sacrificando a los animales enfermos y, además, inactivando por calor la leche comercializada (pasteurización). La implementación de estas medidas en los distintos países provocó polémica, resistencia, demoras y considerables gastos, si se considera que, por ejemplo, en Estados Unidos se estimaba que 4\% del ganado era tuberculoso en 1922, cifra que descendió a 0,4\% en $1938^{22}$. En Gran Bretaña se citaba un porcentaje de 17 a18\% de vacunos enfermos, que subía a $40 \%$ al tomar en cuenta solo vacas en lactancia ${ }^{23}$.

Habiéndose reputado como muy frecuente la escrofu- losis hasta el siglo XIX, en el siglo XX la linfoadenitis tuberculosa tendía a disminuir su frecuencia ${ }^{24}$. En realidad, el descenso se apreció especialmente después de 1950 cuando en varios países se iba extendiendo el control del ganado y la pasteurización de la leche. La linfoadenitis tuberculosa pasó a constituir 5 a 10\% de las tuberculosis activas en países desarrollados, manteniendo una frecuencia de hasta $44 \%$ en grupos de inmigrantes ${ }^{25}$.

En 1974 un estudio epidemiológico en Suecia demostró una relación inversa entre la prevalencia de tuberculosis en el ganado y la mortalidad humana por tuberculosis. Sugería que la infección por bacilo bovino en la niñez podía tener un efecto protector contra la infección tuberculosa en adultos, particularmente pulmonar ${ }^{26}$. Esto podría explicar la estimación de una alta frecuencia de escrófula frente a una aparente menor frecuencia de tuberculosis pulmonar en siglos pasados. Concordaba con la observación clínica de Antoine Marfan, quien en 1886 comunicó que individuos con cicatrices de escrófula sufrida en la infancia desarrollaban muy infrecuentemente una tuberculosis pulmonar: sugirió que las escrófulas infantiles eran, en general, tuberculosis atenuadas que tras su curación dejaban inmunidad contra la tuberculosis pulmonar ${ }^{27}$.

\section{Micobacterias no tuberculosas}

Desde principios del siglo XX se comunicó el hallazgo de bacilos ácido-alcohol resistentes en esputo y lesiones purulentas cutáneas: sus caracteres en los cultivos y los resultados obtenidos al inocularlos en animales de laboratorio eran diferentes a los comprobados con el bacilo tuberculoso. Se las empezó a designar micobacterias no tuberculosas, entre otras denominaciones ${ }^{28}$. Hacia mediados de siglo se discutía el rol de esos bacilos en la patogenia de las lesiones de las cuales se habían aislado, especialmente en casos de afecciones pulmonares. En 1954 Tampe y Runyon, acogían la hipótesis de que en algunos casos estaban íntimamente asociadas con enfermedad pulmonar de tipo tuberculoso sin que pudiera demostrarse la presencia de M. tuberculosis. Propusieron una tentativa ordenación en grupos de las micobacterias no tuberculosas ${ }^{29}$. Esta fue ampliada en una posterior clasificación en cuatro grupos, que abarcaban cada uno distintas especies ${ }^{30}$.

El espectro de micobacterias patógenas para el ser humano ha quedado integrado -aparte de $M$. leprae- en dos grandes conjuntos. Uno es el complejo de $M$. tuberculosis al que pertenecen especies que reconocidamente causan enfermedad tuberculosa: incluye, entre otros, $M$. tuberculosis, M. bovis y M. africanum. El otro conjunto es el de las micobacterias no tuberculosas, también llamadas atípicas, que actualmente abarca alrededor de 150 especies $^{31}$. Para tipificarlas, se requieren laboratorios 
que dispongan de compleja tecnología microbiológica y molecular ${ }^{32,33}$.

Las micobacterias no tuberculosas son bacilos de vida libre que se encuentran en el agua, tierra, animales, material vegetal o alimentos contaminados; de ahí que algunos autores prefieran el nombre de micobacterias ambientales. En condiciones habituales no son patógenas; pero eventualmente pueden causar una variedad de síndromes clínicos, no solo en pacientes inmunocomprometidos, sino en hospederos inmunocompetentes ${ }^{34,35}$.

\section{Linfoadenitis cervico-facial por micobacterias no tuberculosas}

En 1956 y1957 se publicaron casos de niños con linfoadenitis cervical y cervico-facial por micobacterias no tuberculosas. En material obtenido por punción o extirpación se observaban bacilos ácido-alcohol resistentes, y las biopsias que se examinaron presentaban necrosis caseosa y granulomas epiteloideos. Los cultivos mostraron micobacterias no tuberculosas, para las cuales se sugirió el nombre de Mycobacterium scrofulaceum en la primera serie y se consideraron "saprófitas" en la segunda ${ }^{36,37}$.

En 1972 Lincoln y Gilbert presentaron una revisión de casos publicados de enfermedades por micobacterias no tuberculosas en menores de 17 años. Además del cultivo bacteriano, consideraron útil la aplicación de algunos de los siguientes criterios para caracterizar los casos de linfoadenitis: niños de uno a cuatro años, sin historia de contacto tuberculoso, radiografía pulmonar negativa, sin respuesta apreciable a fármacos antituberculosos, supuración precoz, compromiso ganglionar unilateral y de glándula salival ${ }^{38}$.

El hallazgo de granulomas epiteloideos caseificantes y bacilos ácido-alcohol resistentes en la biopsia ganglionar orienta hacia una etiología micobacteriana. Si además se observan microabscesos, granulomas poco definidos o no caseificantes y escasas células gigantes, puede sugerirse -pero no asegurarse- que se trata de una micobacteria no tuberculosa ${ }^{39}$.

La linfoadenitis cervical o cervico-facial es la localización más frecuente de las enfermedades asociadas a micobacterias no tuberculosas. Afecta principalmente a niños entre 2 y 4 años. Se presenta como una tumefacción indolora, generalmente unilateral, de ganglios yugulodigástricos, submentonianos, parotídeos o preauriculares. Puede evolucionar a la cicatrización y calcificación o aumentar de volumen con signos inflamatorios, reblandecimiento y fistulización ${ }^{40-44}$. Entre las especies más frecuentemente identificadas se encuentran $M$. avium, $M$. scrofulaceum, M. kansasii, M. lentiflavum y M. haemophilum, aunque hay variaciones geográficas ${ }^{31,41}$. Dada la distribución de los grupos ganglionares más afectados y considerando que los niños pequeños tienen mayor exposición a materias del suelo, se sugiere que las principales vías de contagio son la mucosa bucal y faríngea, piel, conjuntiva y glándulas salivales ${ }^{45}$.

\section{La escrófula ayer y hoy}

En la medicina del ámbito hipocrático se explicaba la escrófula como el exceso del humor flema, que se acumulaba en glándulas esponjosas del cuello. En el siglo XVII se propuso que, a consecuencia de la acrimonia del componente líquido de la sangre, este se acumulaba en ganglios y pulmones, que tenían la propiedad de hincharse y endurecerse. Se establecía así una relación de la tisis pulmonar con la escrófula, sugiriéndose la existencia de la entidad "tisis escrofulosa". En la centuria siguiente se notó a ojo desnudo el aspecto caseoso similar de ambas lesiones $^{46}$. Esto después se corroboró en el examen microscópico con la descripción de necrosis y minúsculos granulomas epiteloideos denominados tubérculos ${ }^{1}$.

El desarrollo de la microbiología puso en primer lugar la etiología infecciosa de las linfoadenitis cervicales, llegándose después a conocer decenas de agentes de afecciones agudas, subagudas o crónicas ${ }^{47}$. Entre ellas, la mayoría de las lesiones ganglionares granulomatosas necrosantes eran causadas por M. tuberculosis y M. bovis. Al manifestarse una tendencia a la disminución de la tuberculosis humana y del ganado vacuno, y extenderse la práctica de basar los diagnósticos en los resultados del cultivo y no solo en la visualización de bacilos ácidoalcohol resistentes, fue aumentando el hallazgo de casos con compromiso ganglionar cervico-facial por micobacterias atípicas o ambientales. Esta sucesión determinó que el nombre de escrófula fuera reemplazado por el de linfoadenitis o linfoadenopatía tuberculosa y después por linfoadenitis micobacteriana.

Grange y cols., reconocieron tres patrones clínicos de distinta etiopatogenia en el conjunto de casos con linfoadenitis de tipo tuberculoso. Un primer grupo con compromiso de los ganglios tonsilares en niños pequeños por ingestión de leche contaminada con cepas bovinas, en franca disminución. Un segundo grupo con afectación de ganglios cervicales en niños pequeños por especies ambientales de micobacterias. El tercer grupo corresponde a una lesión de cualquier grupo ganglionar, aunque preferentemente supraclavicular, de niños mayores y adultos por cepas humanas $\mathrm{y}$, con menor frecuencia, por cepas bovinas $^{48}$.

Un centro de referencia inglés publicó en 1992 el estudio bacteriológico de muestras de linfoadenopatía micobacteriana de 1.817 pacientes de diversas edades, mayoritariamente de grupo étnico subcontinental indio. El 75,9\% correspondía a ganglios cervicales. Se iden- 
tificó $M$. tuberculosis en 1.677 casos, M. bovis en 25 , $M$. africanum en 16 y micobacterias no tuberculosas o ambientales en $94^{48}$. En comparación con los datos que el mismo laboratorio había obtenido el decenio anterior, apreciaron disminución de la linfoadenitis por $M$. tuberculosis y un aumento de la frecuencia y variedad de micobacterias ambientales ${ }^{49}$.

En Estados Unidos, Margileth mostró que en 168 niños con linfoadenitis cervico-facial de tipo tuberculoso, la etiología correspondió a micobacterias no tuberculosas en $89,2 \%$ y a $M$. tuberculosis en $10,7 \%{ }^{47}$.

Según los caracteres epidemiológicos de la linfoadenitis periférica, Fontanilla y cols., comunicaron la siguiente comparación entre las causadas por micobacterias del complejo tuberculoso y las causadas por micobacterias no tuberculosas: las tuberculosas predominan en personas de 20 a 40 años de países con tuberculosis endémica, son comunes en países con endemia de infección por $\mathrm{VIH}$, infrecuentes en países desarrollados, y se localizan en ganglios cervicales. Las no tuberculosas tienden a presentarse en países sin endemia de tuberculosis, infrecuentemente asociadas con VIH, de localización cervico-facial. En países desarrollados donde no hay endemia de tuberculosis más de $60 \%$ de los enfermos de linfoadenitis tuberculosa son inmigrantes ${ }^{50}$. En el siglo XX pareció pasar a un segundo plano la importancia atribuida antaño a la predisposición personal a la escrófula tipificada por la "constitución diatésica". Sin embargo, se ha avanzado en identificar condiciones que pueden determinar que algunas personas sean susceptibles o resistentes a la infección por micobacterias. Al respecto se puede mencionar el reconocimiento de mecanismos de inmunidad local o sistémica normal o alterada, y en particular la identificación de mutaciones autosómicas o ligadas al sexo que confieren susceptibilidad a infecciones micobacterianas diseminadas ${ }^{51-55}$.

Agradecimientos. Las microfotografías que ilustran esta nota histórica pertenecen a la colección del Servicio de Anatomía Patológica del Hospital de Niños Roberto del Río, Santiago, Chile. Fueron gentilmente facilitadas por el Dr. Luis Velozo Papez, Jefe del Servicio.

\section{Resumen}

En el siglo XIX se pensaba que la tuberculosis y la tumefacción ganglionar cervical llamada escrófula afectaban a individuos predispuestos por una "constitución diatésica" heredada. En 1882 Robert Koch demostró que lesiones tuberculosas y escrofulosas humanas eran causadas por el bacilo Mycobacterium tuberculosis. A principios del siglo XX se estableció que Mycobacterium bovis, bacilo de la tuberculosis del ganado, podía también causar linfoadenitis cervical en humanos, especialmente en niños, por la ingestión de leche de vacas enfermas. La condición disminuyó después que se controló la infección en el ganado y se introdujo la pasteurización de la leche. En 1956 se describió la linfoadenitis cervicofacial granulomatosa necrosante y supurada causada por micobacterias no tuberculosas. Afecta principalmente a niños bajo los cinco años, especialmente en países sin endemia de tuberculosis. Las linfoadenitis cervicales tuberculosas predominan en adultos jóvenes en países con tuberculosis endémica y en individuos infectados por VIH.

\section{Referencias bibliográficas}

1.- Duarte I. La escrófula en el siglo XIX. Rev Chilena Infectol 2017; 34: 55-9.

2.- Koch R. The aetiology of tuberculosis. Am Rev Tuberc 1932; 25: 285-323. Traducción del alemán de Berna y Max Pinner del artículo publicado en Berliner klinische Wochenschrift 1882; XIX, 221.

3.- Brock T D. Robert Koch. A life in Medicine and Bacteriology. Madison, Wisconsin USA: Science Tech Publishers; 1988, p 126-37.

4.- Orth J. Compendio técnico de Anatomía e Histología Patológicas. Traducido al castellano de la tercera edición alemana (1884) por Francisco Fonck y Eduardo Hoffman. Santiago, Chile: Imprenta de la Unión; 1886, p. 36-7.

5.- Ledermann W. La tuberculosis después del descubrimiento de Koch. Rev Chilena Infectol. Edición Aniversario 2003: 48-50.

6.- Trudeau E L. An autobiography. Philadelphia: Lea \& Febiger; 1916.

7.- Knopf SA. Dr. Edward Livingston Trudeau. In memoriam. Brit J Tuberculosis 1916; 10: 80-5.

8.- Roberts C S. Trudeau at Lake Saranac. Walker HK, Hall WD, Hurst JW, editors. Clinical Methods: The History, Physical, and Laboratory Examinations, $3^{\text {rd }}$ ed. Boston: Butterworths Publishers; 1990, p.191-2.

9.- Schride H. Órganos hematopoyéticos. Achoff L. Tratado de Anatomía Patológica. Tomo II. Traducción de Julio Sánchez Lucas. Barcelona: Editorial Labor S. A.; 1934, p. 142.

10.- Virchow R. Die Cellularpathologie in ihre Begründung auf physiologische und pathologische Gewebelehre. Berlin: Verlag von August Hirschwald: 1858, p. 419-25.

11.- Klippe H J, Kirsten D, Andrée C. Rudolf Virchow (18211902) und der Ursprung des Begriffes "Granulom". Pneumologie 2004; 58: 449-54.

12.- Ribbert H, Sternberg C. Tratado de Patología General y Anatomía Patológica, $2^{\mathrm{a}}$ edición. Traducción de Francisco Piñero. Barcelona: Editorial Labor S. A.; 1933, p. 275.

13.- Miller F J W, Cashman J M. Origin of peripheral tuberculous lymphadenitis in children. Lancet 1958; 1: 286-9.

14.- Lessel E F Jr. Status of the generic name Mycobacterium Lehmann and Neumann. International Bulletin of Bacteriological Nomenclature and Taxonomy 1960; 10: 4754.

15.- Smith T. Comparative study of bovine tubercle bacilli and of human bacilli from sputum. J Exp Med 1898; 3: 451-511. 
16.- Final report of the Royal Commission on Tuberculosis. Br Med J 1911; 2 (2637): 122-5.

17.- Karlson A G, Lessel E F. Mycobacterium bovis. Nom. Nov. Int J Syst Bacteriology 1970; 20: 273-82.

18.- Griffith A S, Munro W T. Human pulmonary tuberculosis of bovine origin in Great Britain. J Hyg (Lond) 1944; 43: 22940.

19.- Griffith A S. Bovine tuberculosis in man. Tubercle 1937; 18: 529-43.

20.- Rich A R. The pathogenesis of tuberculosis. Springfield, Illinois: Charles C. Thomas; 1944, p 58.

21.- Raw N. The control of bovine tuberculosis in man. London: Balliere, Tindal \& Cox. London; 1937, p 22.

22.- Dormandy T. The white death: a history of tuberculosis Capítulo 29: Milk. London: The Hambledon Press; 1999, p. 329-38.

23.- Francis J. Control of infection with the bovine tubercle bacillus. Lancet 1950; i: 34-9.

24.- Aschoff L. Anatomía Patológica de la tuberculosis: conferencias pronunciadas en la Universidad de Verano de Santander. Traducción de Enrique Oliva. Barcelona: Editorial Labor S.A; 1935.

25.- Grzybowski S, Allen E A. History and importance of scrofula. Lancet 1995; 346 (8988): 1472-4.

26.- Sjögren I, Sutherland I. Studies of tuberculosis in men in relation to infection in cattle. Tubercle 1974; 56: 113-27.

27.- Marfan A. De l'immunité conférée par la guérison d'une tuberculose locale pur la phthisie pulmonaire. Archives Generales de Médecine 1886; 57: 575-9.

28.- Wolinski E. Nontuberculous mycobacteria and associated diseases. Am Rev Respir Dis 1979; 119: 107-59.

29.- Tampe A, Runyon E H. The relationship of "atypical" acid fast bacteria to human disease: a preliminary report. J Lab Clin Med 1954; 44: 202-9.

30.- Runyon EH. Anonymous mycobacteria in pulmonary disease. Med Clin North Am 1959; 43: 273-90.

31.- Núñez Cuadros E, Baquer Artigao F. Recomendaciones de la Sociedad Española de Infectología Pediátrica sobre el diagnóstico y tratamiento de las adenitis por micobacterias no tuberculosas. An Pediatr (Barc) 2012; 77: 208.e1-208. e12.

32.- Griffith D E, Aksamit T, Brown-Elliot B A, Catanzaro A, Daley C, Gordin F, et al. An official ATS/IDSA statement: diagnosis, treatment, and prevention of non-tuberculous mycobacterial diseases. Am J Respir Crit Care Med 2007; 175: 367-416.

33.- Tortoli E. The new mycobacteria: an update. FEMS Immunol Med Microbiol 2006; 48: 159-78.

34.- Ruiz Manzano J, Manterola J M, Ausina V, Sauret J. Nomenclatura y clasificación de las micobacterias. Arch Bronconeumol 1998; 34: 154-7.

35.- Jarzembowski J A, Young M B. Nontuberculous mycobacterial infections. Arch Pathol Lab Med 2008; 132: 1333-41.

36.- Masson A M, Prissick F H. Cervical lymphadenitis in children caused by chromogenic Mycobacteria. Can Med
Assoc J 1956; 75: 798-803.

37.- Keith H M, Weed L A, Needham G M. Non-tuberculous acid-fast bacilli in cervical adenitis. Pediatrics 1957; 20: 688-97.

38.- Lincoln E M, Gilbert L A. Disease in children due to mycobacteria other tan Mycobacterium tuberculosis. Am Rev Respir Dis 1972; 105: 683-714.

39.- Kraus M, Benharroch D, Kaplan D, Sion-Vardy N, Leiberman A, Dima H, et al. Mycobacterial cervical lymphadenitis: the histological features of non-tuberculous mycobacterial infection. Histopathology 1999; 35: 534-8.

40.- Appling D, Miller R H. Mycobacterial cervical lymphadenopathy: 1981 update. Laryngoscope 1981; 91: 1259-66.

41.- Wolinsky E. Mycobacterial lymphadenitis in children: a prospective study of 105 nontuberculous cases with longterm follow up. Clin Infect Dis 1995; 20: 954-63.

42.- Newcombe J F. Tuberculous cervical lymphadenopathy. Postgrad Med J 1971; 47: 713-7.

43.- Ord R J, Matz G J. Tuberculous cervical lymphadenitis. Arch Otolaryngol 1974: 99: 327-9.

44.- López-Varela E, García-Basteiro A L, Santiago B, Warner D, van Ingen J, Kampmann B. Non-tuberculous mycobacteria in children: mudding the waters of tuberculous diagnosis. Lancet Respir Med 2015; 3: 244-56.

45.- Panesar J, Higgins K, Daya H, Forte V, Allen U. Nontuberculous mycobacterial cervical adenitis: a ten-year retrospective review. Laryngoscope 2003; 113: 149-54.

46.- Duarte I, Chuaqui C. Historia de la escrófula: de la discrasia humoral a la consunción. Rev Med Chile 2016; 44: 503-7.

47.- Margileth A M. Cervical adenitis. Pediatr Rev 1985; 7: 1324.

48.- Grange J, Collins C, Yates M. Bacteriological survey of tuberculous lymphadenitis in south-east England: 1973-80. J Epidemiol Community Health 1982; 36: 157-61.

49.- Yates M D, Grange J M. Bacteriological survey of tuberculous lymphadenitis in southeast England, 1981-1989. J Epidemiol Community Health 1992; 46: 332-5.

50.- Fontanilla J M, Barnes A, von Reyn C F. Current diagnosis and management of peripheral tuberculous lymphadenitis. Clin Infect Dis 2011; 53: 555-62.

51.- Palmer C E, Long M W. Effects of infection with atypical mycobacteria on BCG vaccination and tuberculosis. Am Rev Respir Dis 1966; 94: 553-68.

52.- Patel S Y, Doffinger R, Barcenas-Morales G, Kumararatne D S. Genetically determined susceptibility to mycobacterial infection. J Clin Pathol 2008; 61: 1006-12.

53.- Casanova J L, Abel L. Genetic dissection of immunity to mycobacteria: the human model. Annu Rev Immunol 2002; 20: 581-620.

54.- Qu H Q, Fischer-Hoch S P, McCormick J B. Molecular immunity to mycobacteria: knowledge from the mutation and phenotype spectrum analysis of Mendelian susceptibility to mycobacterial diseases. Int J Infect Dis 2011; 15: e305-13.

55.- $\mathrm{Wu} \mathrm{U} \mathrm{1,} \mathrm{Holland} \mathrm{S} \mathrm{M.} \mathrm{Host} \mathrm{susceptibility} \mathrm{to} \mathrm{non-tuberculous}$ mycobacterial infections. Lancet Infect Dis 2015; 15: 96880 . 